\title{
Mechanical model for Inverted arch of highway tunnel in weak surrounding rock and dynamic design

\author{
Zhongming SU
}

Shanxi Transportation Research Institute, Key Laboratory of Highway Construction \&Maintenance
Technology in Loess Region, Ministry of Transport,Taiyuan,030006, china

email:375216207@qq.com

\begin{abstract}
Keywords: Weak Surrounding Rock. Shallow Buried. Inverted Arch. Three-hinged Arch. Dynamic Design
\end{abstract}

\begin{abstract}
Due to the lack of theoretical basis for the design of inverted arch, the inverted arch design of many tunnels is not reasonable, and the problem is more prominent in the design of the shallow buried tunnel. By analyzing the relationship between the soft foundation and the stability of tunnel structure, the method of determining the load of shallow buried tunnel is presented. With the construction technology of the inverted arch, the mechanical model of the three- hinged inverted arch is established, and the formula for calculating the reasonable arch axis and the internal force calculation formula are given. The design procedure of inverted arch in shallow buried tunnel is presented, and the idea of dynamic design is introduced into the design of the inverted arch.
\end{abstract}

\section{Introduction}

Along with the rapid development of highway, the engineering geological conditions of the tunnel are more and more complex. A large number of inverted arch structures occurred in the construction of weak surrounding rock tunnel. Because of the current " codes for highway tunnel design " [1]and "Guidelines for design of highway tunnel "[2]do not give the method of determining the load of inverted arch, and also do not indicate the mechanical model for inverted arch and in the design of inverted arch, the structural form and design parameters are selected only by experience.

The design of the inverted arch is not reasonable, which not only increases the cost of the project, but also increases the height of the tunnel excavation, such problem is particularly prominent in the construction of shallow buried tunnel [3-4].The design mechanical model and design method of inverted arch in the shallow buried weak surrounding rock tunnel are discussed in this paper.

\section{Factor of shallow buried tunnel inverted arch load}

According to the stability of surrounding rock and supporting structure, the load of shallow buried tunnel after excavation and support can be divided into two situations: one is the overall sinking of the whole structure, the other is the non-overall sinking of the whole structure. In the first case, the vertical pressure of top layer of the tunnel is born by the soft rock as the base of the inverted arch. At this time, if the uniform load of top layer of the tunnel is $q$, the inverted arch subject to the uniform force (upward load) is $p=q$. This model is called the inverted arch load model with overall sinking, which has been used in some projects, but it is rare. In the second case, the vertical pressure of top layer of the tunnel is balanced by the friction force at the sides of the tunnel (including the force of the bolt) and the bottom of force of support (lining).

At this time, if we want to determine the bearing load to the inverted arch of the soft rock basis, firstly we need to determine the friction on both sides. The determination of friction is very complex, but it can be estimated by the surrounding rock and the support. At this time, if the uniform load of top layer of the tunnel is $q$, the inverted arch subject to the uniform force (upward load) is $p=c q . c$ is coefficient of the inverted arch bearing the load from top layer of the tunnel, referred to as the load factor, which is determined by the surrounding rock and the support. For example, in the weak surrounding rock $c=0.8$, in the good surrounding rock $c=0.5$. This model is called the inverted arch load model with non-overall sinking, which occurred in a large number of 
projects.

\section{Shallow buried tunnel inverted arch load}

The basic basis of the design of the inverted arch is the load during the service period. Therefore, the reasonable determination of the inverted arch load is the key to the design of the inverted arch. In general, the load of the inverted arch is closely related to the upper load of the tunnel. Since the upper load of deep buried tunnel is still in the stage of experience judgment, so the inverted arch load of deep buried tunnel is not considered. In the light of the upper structure load of the shallow tunnel, the determination method of the inverted arch load of the shallow tunnel is established.

(1) (Buried depth) $H \leq h_{q}$ (Equivalent load height)

$q=\gamma H$, where $q$ is the vertical uniform pressure $\left(k N / \mathrm{m}^{2}\right), \gamma$ is the bulk density of the surrounding $\operatorname{rock}\left(\mathrm{kN} / \mathrm{m}^{3}\right), H$ is the buried depth of tunnel(m).

(2) (Equivalent load height) $h_{q}<H$ (Buried depth) $\leq H_{p}$ (Critical depth)

$q=\gamma H\left(1-\lambda H \tan \theta / B_{t}\right)$, where $B_{t}$ is the tunnel excavation span; $\lambda$ is the lateral pressure coefficient, $\lambda=\frac{\tan \beta-\tan \varphi_{c}}{\tan \beta\left[1+\tan \beta\left(\tan \varphi_{c}-\tan \theta\right)+\tan \varphi_{c} \tan \theta\right]} ; \theta$ is the friction angle of failure surface of both sides; when no field references, it can be adopted according to the following Table1 and $\beta$ is the failure angle of maximum thrust, $\tan \beta=\tan \varphi_{\mathrm{c}}+\sqrt{\frac{\left(\tan ^{2} \varphi_{c}+1\right) \tan \varphi_{c}}{\tan \varphi_{c}-\tan \theta}}$.

Table1 $\theta$ value in different surrounding rock

\begin{tabular}{|c|c|c|c|c|}
\hline surrounding rock level & I \& II \&III & IV & V & VI \\
\hline$\theta$ & $0.9 \varphi_{c}$ & $(0.7 \sim 0.9) \varphi_{c}$ & $(0.5 \sim 0.7) \varphi_{c}$ & $(0.3 \sim 0.5) \varphi_{c}$ \\
\hline
\end{tabular}

\section{Internal force analysis of inverted arch}

Taking the middle line of the tunnel as the boundary, both sides of the inverted arch is not often constructed at the same time, so the midline of the inverted arch is the weak position in the construction. So it is reasonable to take the inverted arch as the three-hinged arch. The reasonable axis equation of inverted arch can be expressed by the following formula [5]:

$$
y=\frac{4 f}{l^{2}} x(1-x)
$$

By establishing the force balance equation, the internal force of the three-hinged arch can be obtained as follows.

$$
\begin{aligned}
& M=0 \\
& \left.N=\frac{p l^{2}}{8 f} \sqrt{1+\left(\frac{4 f(l-2 x)}{l^{2}}\right)^{2}}\right\} \\
& Q=0
\end{aligned}
$$

$$
N_{\max }=\frac{p l^{2}}{8 f} \sqrt{1+\left(\frac{4 f}{l}\right)^{2}}
$$

Where $M$ is the moment $(k N . m), N$ is the axial force $(k N)$,and $Q$ is shear force on vertical section of axis $(k N)$.

Obviously, the inverted arch is in a one-way pressure state. The maximum and minimum values of the axial force and the positions should be determined as for the design of the inverted arch. It can be shown that the maximum axial force appears at the position of the inverted arch foot, of which the value can be calculated by the formula(3), and that the minimum axial force appears at the middle position of inverted arch, of which the value can be calculated by the formula (4). 


$$
N_{\text {min }}=\frac{p l^{2}}{8 f}
$$

$$
\frac{d N_{\max }}{d f}=-\frac{p l^{2}}{8 f} \frac{1}{\sqrt{1+\left(\frac{4 f}{l}\right)^{2}}}
$$

The arch height $f$ of the inverted arch is also an important design parameter. According to the following formula (5), $N_{\max }$ is a monotonically decreasing function of $f$. For tunnels, it is obviously not possible to increase the arch height in order to reduce the axial force of the inverted arch.

\section{Design procedures of inverted arch in shallow buried tunnel}

(1) Determining the load set of inverted arch $p$.

(2) Determining the width of inverted $\operatorname{arch} l$, which can be considered as the maximum tunnel excavation.

(3) Selection of height-width ratio of inverted $\operatorname{arch} r$, in general, $r=1 / 20 \sim 1 / 10$ and the height of the inverted arch can be calculated by $f=r . l$.

(4) In turn, $x=l / 8, l / 4,3 l / 8$ can be put in the formula of reasonable arch axis of the three-hinged arch ,then reasonable construction line elevation of the arch axis can be obtained.

(5) According to the formula of (3)\&(4), the maximum and minimum of axial force in inverted arch can be calculated.

(6) According to the selected materials, the design strength of inverted arch concrete $[\sigma]$ can be selected.

(7) Determining the thickness of inverted arch $d$.The inverted arch can be adopted in two ways, such as uniform thickness and non-uniform thickness. When adopted in uniform thickness, the maximum axial force in the location of inverted arch foot can be considered as the controlling load, and the thickness of inverted arch can be calculated by the following formula:

$$
d=N_{\max } /[\sigma]
$$

When adopted in non-uniform thickness, the middle thickness of inverted arch is the reference thickness and the middle thickness can be calculated by the following formula:

$$
d=N_{\min } /[\sigma]
$$

Then the thickness of inverted arch foot can be determined by the following formula:

$$
D=k . d \text {, where } k \text { is the thickness factor, } k=\sqrt{1+\left(\frac{4 f}{l}\right)^{2}}
$$

(8)Optimizing the inverted arch from the aspects of economy, structure and construction technology, and then complete the design drawings.

(9)Monitoring the stress and deformation of the tunnel after excavation, and observe the rock properties at the bottom of the tunnel, determine whether the previous load set of inverted arch and the assumption of the soft rock base are reasonable or not. If reasonable, then the inverted arch can be constructed by the original design, if not reasonable, the inverted arch should be redesigned according to actual case.

\section{Dynamic design of the inverted arch}

In the process of tunnel excavation, the geological conditions of the actual exposure are often different from that provided by the survey and design stage. Tunnel inverted arch is always but also often after a period of time constructed after the excavation and the support of upper tunnel. The time from the upper support to the excavation of the inverted arch provides the possibility for the optimal design of the inverted arch. The idea of dynamic design and construction monitoring of the new Austrian tunneling method can be introduced into the design and construction of the tunnel inverted arch, so as to make a scientific and rational design. 


\section{Conclusions and suggestions}

(1)The load of inverted arch in shallow buried tunnel can be determined by the load factor and the vertical pressure of top layer of the tunnel

(2)The mechanical state of inverted arch can be designed and analyzed by three-hinged arch, and the inverted arch can be in the axial compression state after the selection of reasonable arch axis, which will be beneficial to the mechanical properties of concrete materials.

(3)The thickness of the inverted arch can be calculated quantitatively, and the thickness of the inverted arch when needed is not less than $20 \mathrm{~cm}$.

(4)The setting of the inverted arch can be determined according to the geological conditions of the tunnel at the bottom and the monitoring results of the tunnel excavation, which can be applied to the design of the inverted arch.

\section{Acknowledgement}

In the process of writing, the paper has been financially sponsored by the application basic research project of Ministry of transportation (NO:2014319771190). Many thanks to the item.

\section{References}

[1] JTG D70- 2004, codes for highway tunnel design[S].

[2] JTG/T D70-2010, Guidelines for design of highway tunnel[S].

[3] Chen Huali, Ren Shangqiang,Hao Leijie. Research on inverted arch in large-span flat tunnel[J].Technology of Highway and Transport,2009(6):16-109.

[4] Guan Baoshu. Key sets of tunnel engineering design[M]. Beijing: China Communications Press,2003.

[5] Long Yuqiu, Bao Shihua, et al. Structural mechanics course[M]. Beijing: Higher Education Press,2003. 